\title{
POSTOPERATIVE CERVICAL SPINAL EPIDURAL HEMATOMAS; TWO CASE REPORTS AND LITERATURE REVIEW
}

\author{
(1) Aykut AKPINAR, (1) Bekir Mahmut KILINÇ, @ Nevhis AKINTÜRK, @ Metin KASAP, @ Tuncer TASÇIOĞLU
}

Istanbul Haseki Training and Research Hospital, Clinic of Neurosurgery, İstanbul, Turkey

Postoperative spinal epidural hematomas are rarely seen and mostly asymptomatic. Urgent surgery is advised in case of the occurrence of neurologic deterioration. Here, we presented two cervical spinal surgery case reports and literature review. In the first case, after anterior cervical microdiscectomy and cage fusion operation, a 35-year-old male patient had paresis because of the cervical epidural hematoma. In the second case, an 85-year-old male patient had multilevel surgery of cervical posterior screw stabilization and posterior laminectomy. In early period, tetraparesis occurred because of large cervicothoracic epidural hemorrhage. The patients' neurologic deficits were improved without surgery.

Keywords: Cervical spinal surgery, postoperative epidural hematoma, paresis

\section{INTRODUCTION}

Acute spinal epidural hematoma can occur as spontaneous, secondary or traumatic $c^{(4)}$. The incidence of spontaneous spinal epidural hematoma is rare (1 case per 1.000.000)(6). Postoperative cervical spinal epidural hematomas are rarely encountered entities ( $0.1 \%$ of all spine cases). Of postoperative spinal epidural hematomas (PSEH) after surgery, asymptomatic ones are extremely common ${ }^{(10)}$, symptomatic ones range from $0.10 \%$ to $0.24 \%$ in all spine surgery group. After spinal surgery, if there is an extra acute neurologic deficit, new computed tomography (CT) and magnetic resonance imaging (MRI) scans should be performed immediately in order to establish a diagnosis and to start treatment. MRI scans can diagnose acute epidural hematoma quickly $(89 \%)^{(10)}$. Rich venous plexus of epidural space may be the origin of the hemorrhage. The area on which epidural hematoma is mostly seen is the thoracic spine $^{(11)}$. After the occurrence of a new neurologic deficit, surgery is suggested. Surgery can solve neurologic deficits in $60 \%$ of patients ${ }^{(10)}$.

\section{CASE REPORTS}

\section{Case 1}

A 35-year-old male patient was admitted to our outpatient clinic with the complaints of left side arm pain and hand numbness. There was no previous history of using anticoagulant, antiaggregant therapy or coagulopathy. No recent trauma was noted. The patient had a previous history of an anterior cervical 5-6 microdiscectomy 3 years ago. He had a new adjacent cervical disc herniation at the cervical 6-7 level. MRI scans revealed cervical 6-7 disc herniation (Figure 1). Anterior microdiscectomy with cage fusion operation was done. Minivac drain was used. In half an hour after the surgery, the patient had mild motor and sensory deficits in his lower and upper extremities. His symptoms progressed quickly and he was soon completely quadriparetic. In the neurological examination, his motor power was reduced (2/5) in both lower and upper extremities, deep tendon reflexes in both lower limbs were increased, the Babinski sign responses were positive, there was hypoesthesia for light touch, and there was hypoesthesia below the cervical 6-7 dermatome.

The complete blood count (hemoglobin, hematocrit, and platelet count), biochemistry profile (kidney function, liver function, proteins, and glucose), prothrombin time, and international normalized ratio (INR) were normal, and he did not have additional comorbidities (hypertension, diabetes mellitus, etc.). His blood was Rh (+). He was a social smoker, smoked approximately 10 boxes per year.

CT scan showed a large cervical epidural bleeding in the anterior region of the spinal cord. The CT scans were not really diagnostically helpful at the operated level. Because of cage fusion, there were metal streak artifacts (Figure 2, 3). MRI revealed anteriorly epidural hematoma compression to the spinal cord extramedullary from C1 to T1 vertebrae levels with isointense or increased signal intensity on T1-weighted image, heterogeneous hyperintense on T2-weighted images (Figure 4-6). 
High-dose steroid treatment was started (bolus $30 \mathrm{mg} / \mathrm{kg}$ administered over 15 minutes with maintenance infusion of $5.4 \mathrm{mg} / \mathrm{kg}$ per hour infused for 23 hours). We planned decompressive surgery and started the preparation of operation theatre. Surprisingly, at the third hour of followup, improvement of paresis in his first lower extremities than upper ones was detected, he recovered and his sensorimotor function was normal. We decided not to operate on the patient, his functions were recovered well after 6 hour. The day after of the operation, his neurologic examination was normal. The patient was discharged three days after the operation.

\section{Case 2}

An 85-year-old male patient had the complaints of pain and numbness on his both arms and hands. The patient had

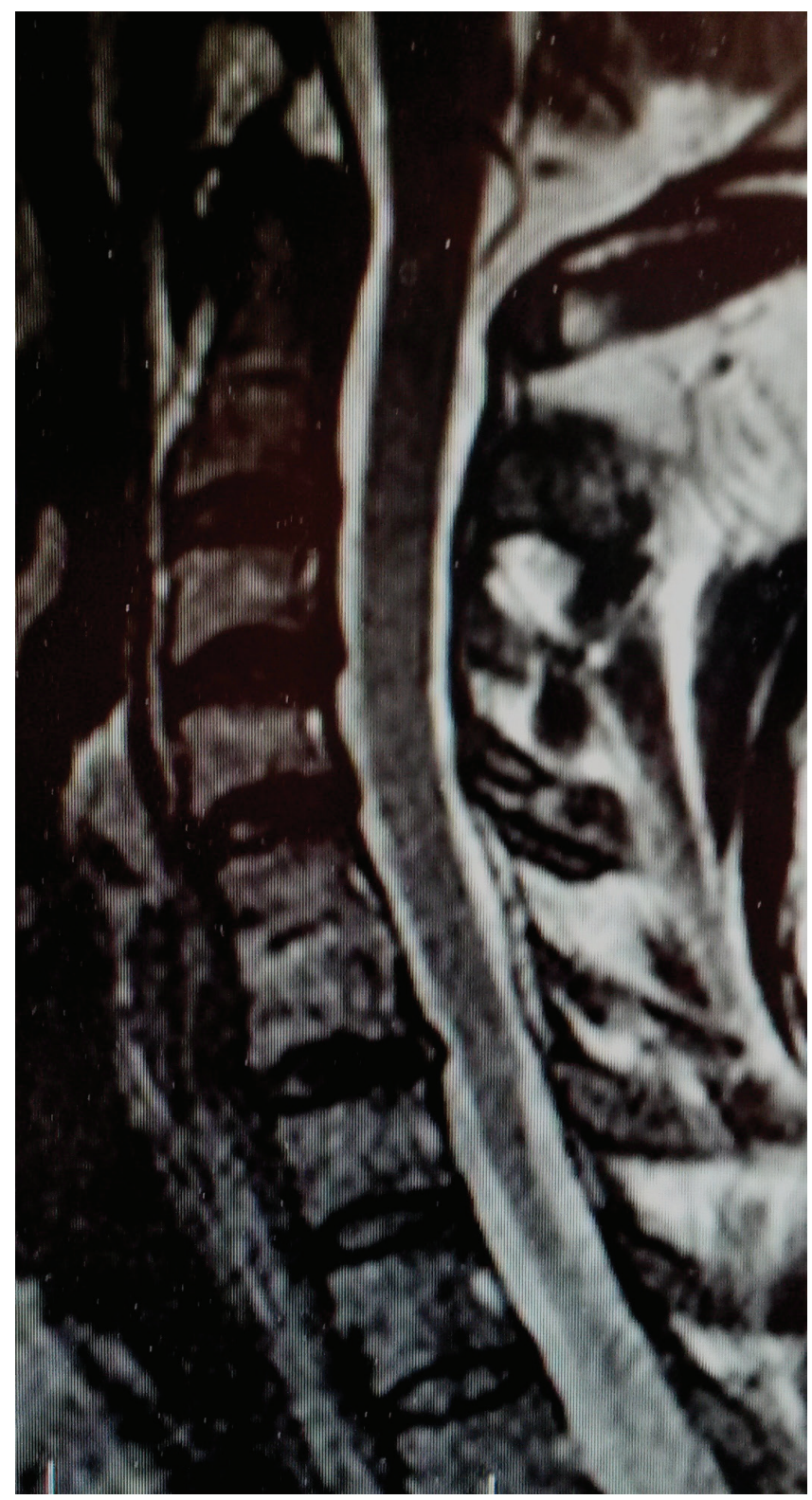

Figure 1. Preoperative sagittal T2-weighted magnetic resonance imaging (cervical 6-7 hernia nucleolus pulposus, cervical 5-6 fusion)

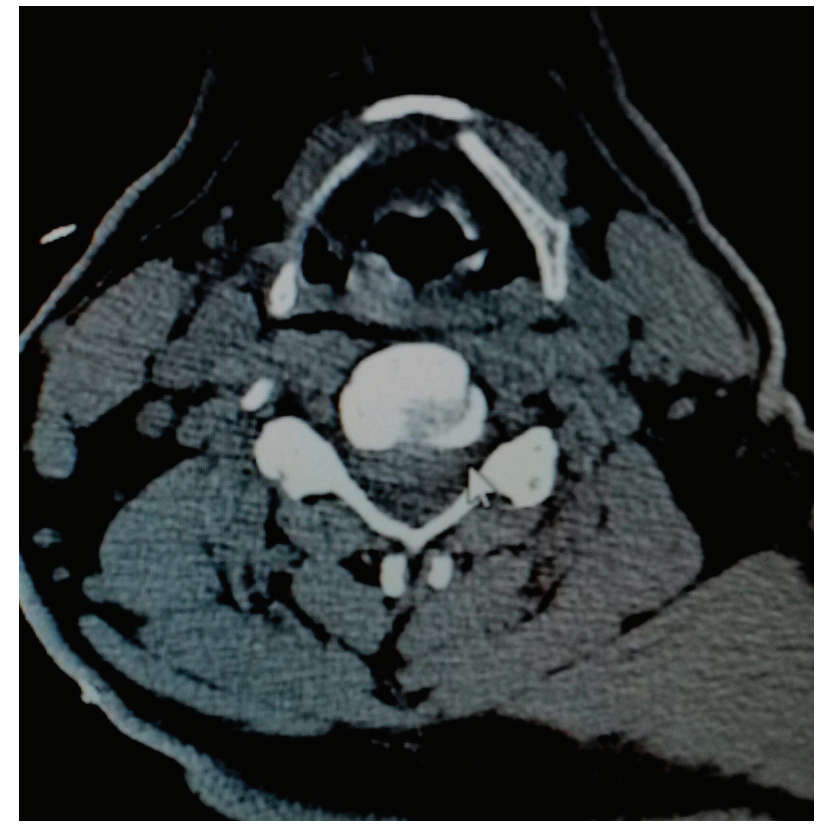

Figure 3. Postoperative axial computed tomography shows the epidural hemorrhage and cord

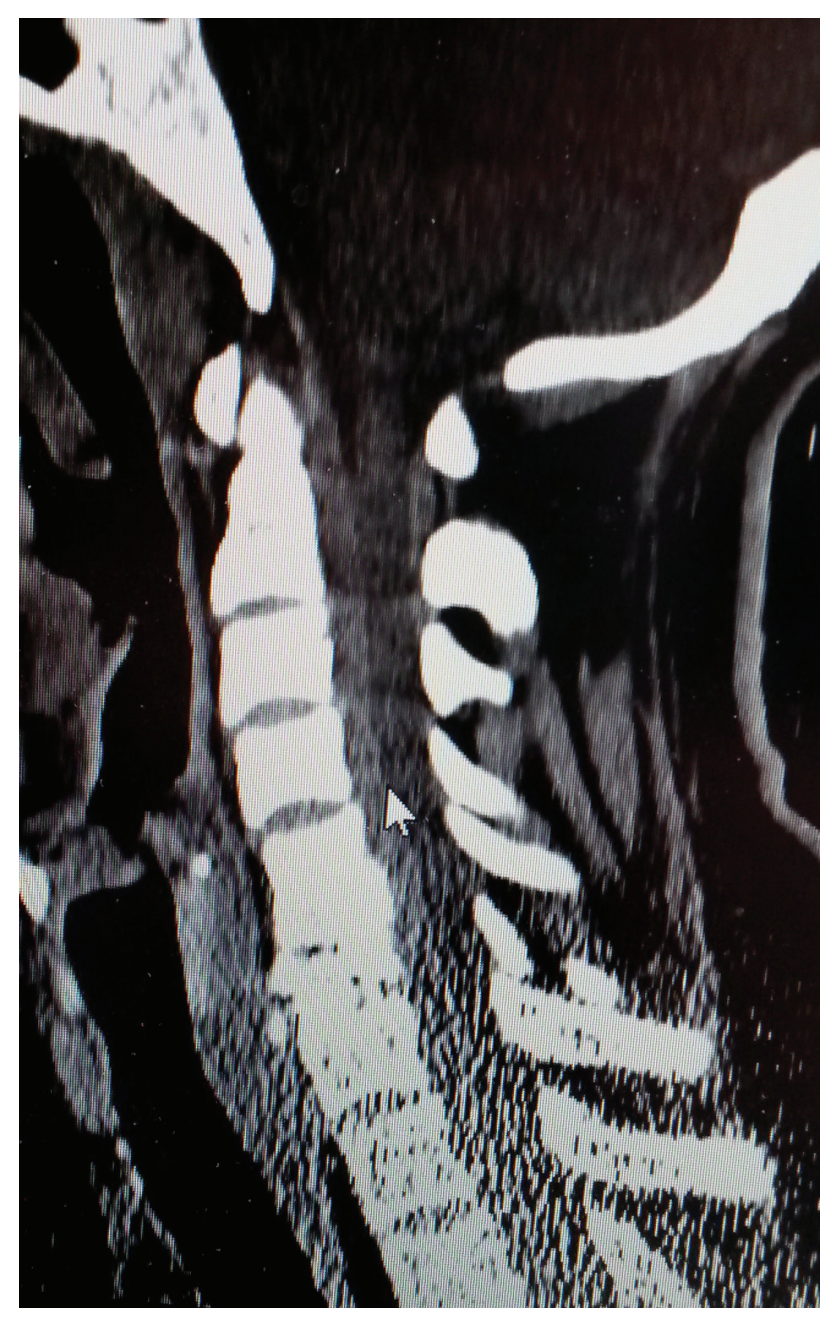

Figure 2. Postoperative sagittal cervical computed tomography (arrow show the epidural hemorrhage and cord line) 
cervical stenosis. The patient underwent anterior cervical 5-67 microdiscectomy and plate, screw operation in an external center one year ago. The patient had a previous history of coronary artery disease, hypertension and by-pass surgery. His blood group was Rh (+) and he was a smoker, smoked 40 boxes/year. He was using acetylsalicylic acid. We stopped acetylsalicylic acid and started low molecular weight heparin during hospitalization. The patient had multilevel surgery of Cervical 2-3-4-5 posterior screw stabilization and cervical 3-4-5 laminectomy posteriorly. The day after the surgery, neurologic deficit was deteriorated and tetraparesis occurred. Motor and sensory deficits in his lower and upper extremities

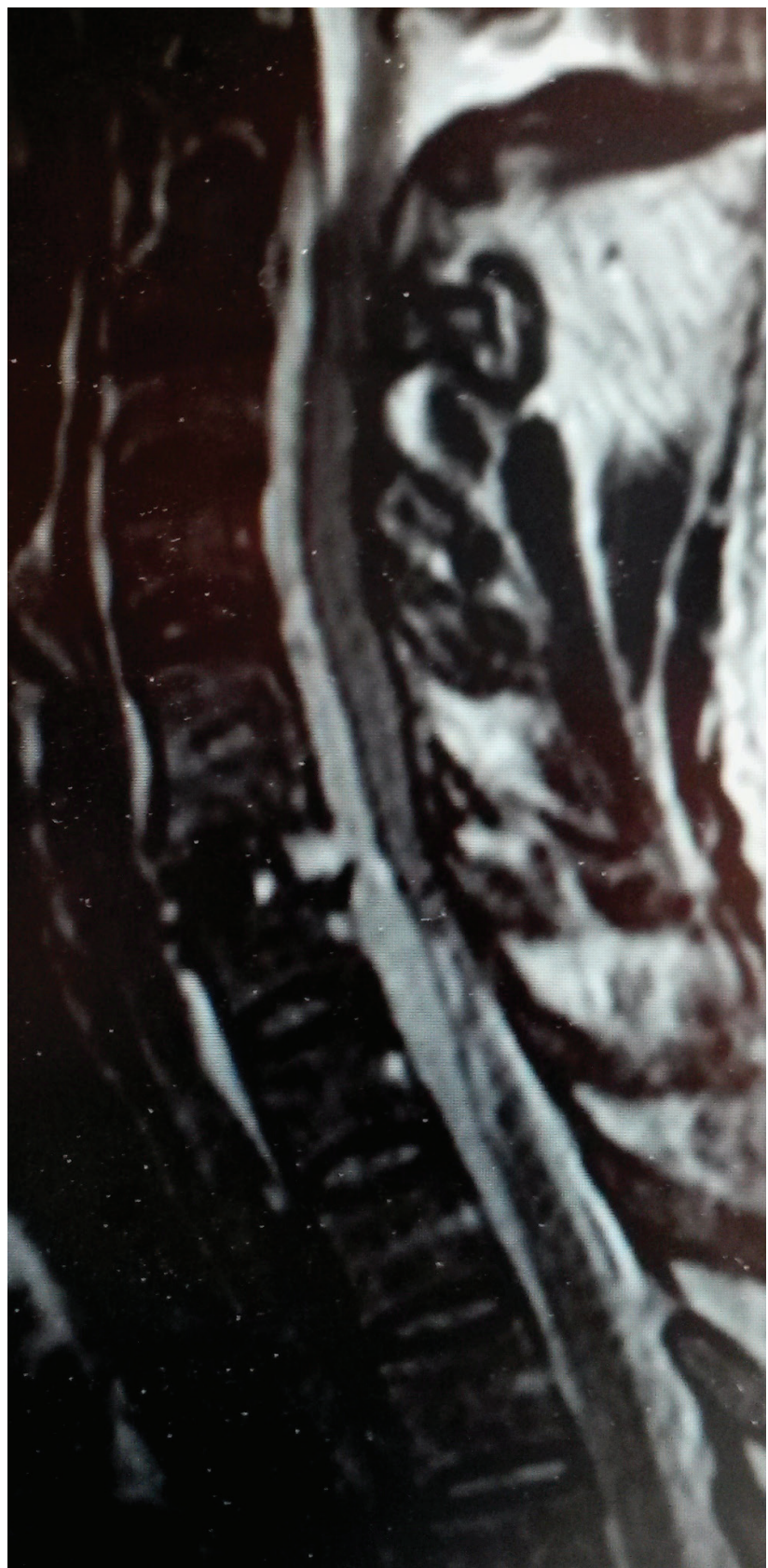

Figure 4. Postoperative sagittal T2-weighted cervical magnetic resonance imaging image shows epidural hemorrhage

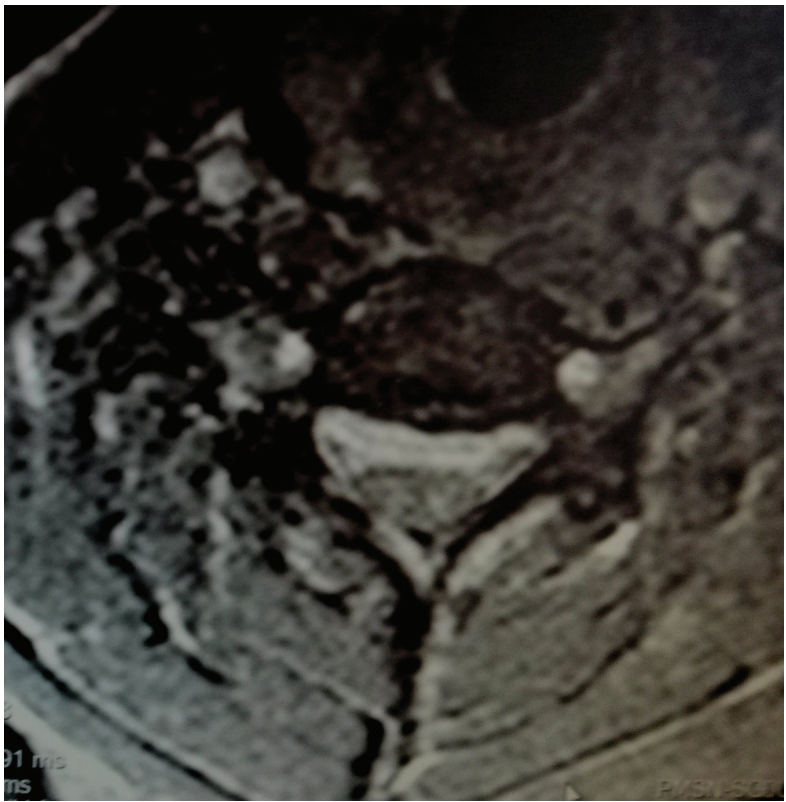

Figure 5. Postoperative axial T2-weighted magnetic resonance imaging

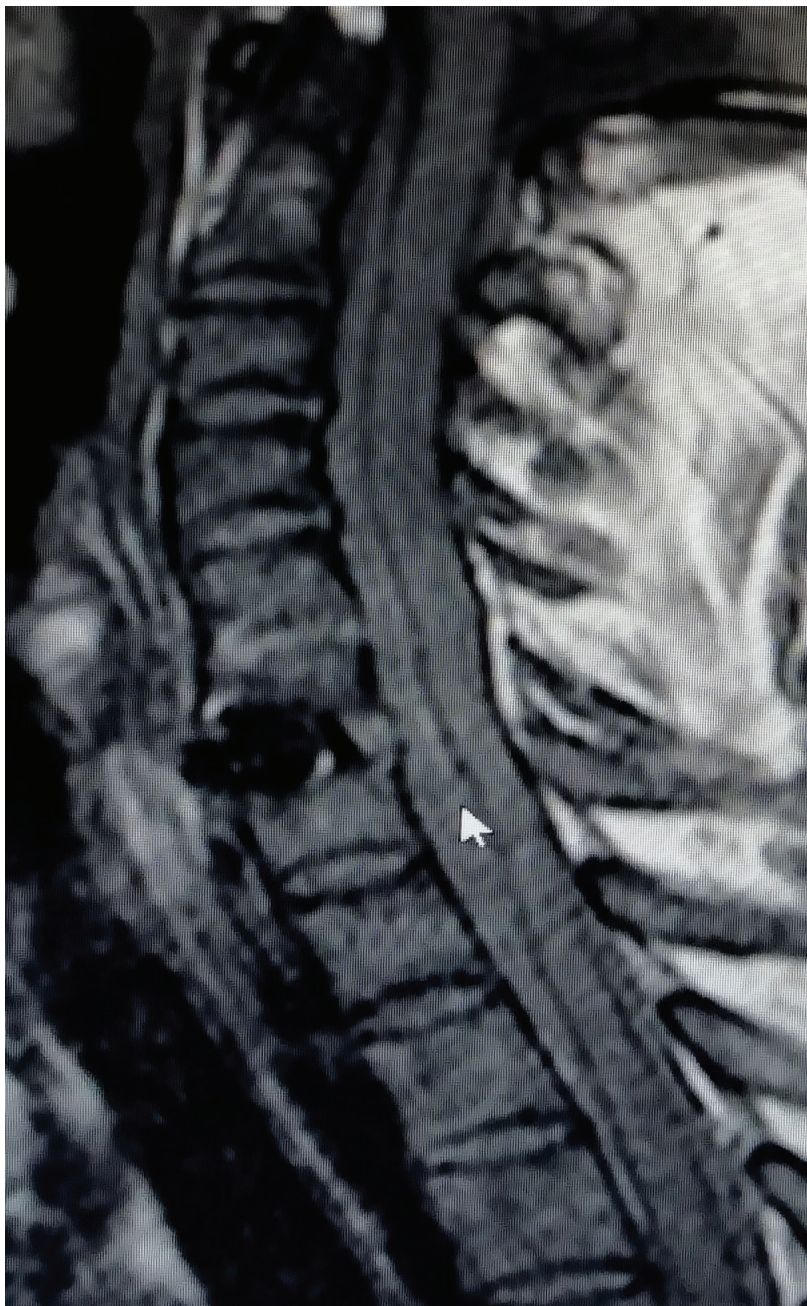

Figure 6. Postoperative sagittal magnetic resonance imaging T1-weighted hem sequence 


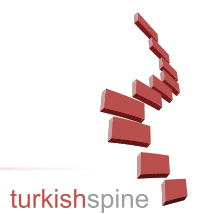

could not resist gravity. In the neurological examination of his motor power, $(2 / 5)$ in both lower and upper extremities, he had ataxia, deep tendon reflexes were hyperactive, bilateral plantar reflexes were dorsal leakage, urinary incontinence, anesthesia for light touch, and hypoesthesia below the cervical

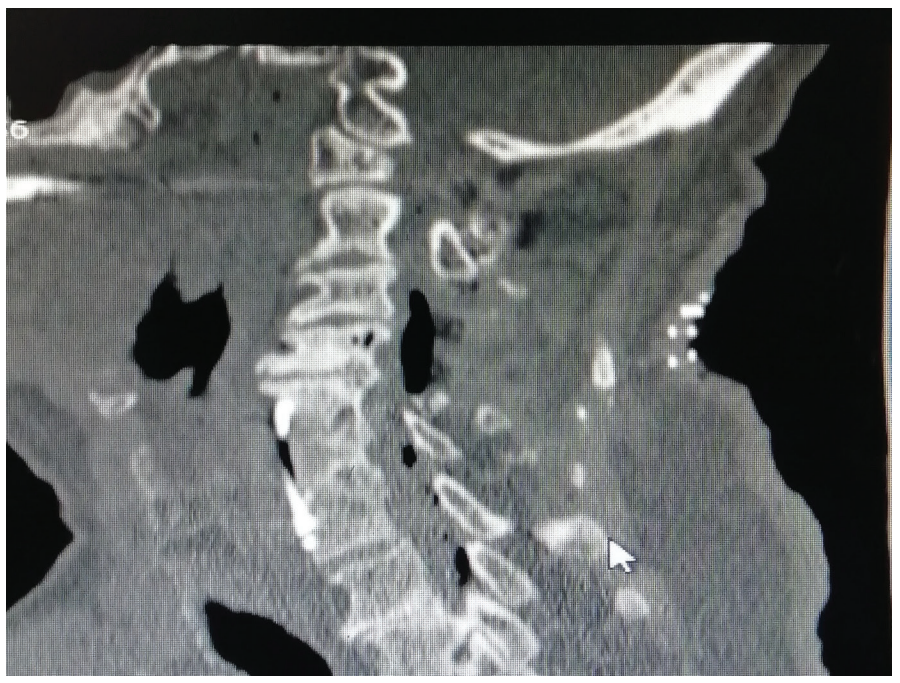

Figure 7. Postoperative Sagittal cervical computed tomography shows air at the region

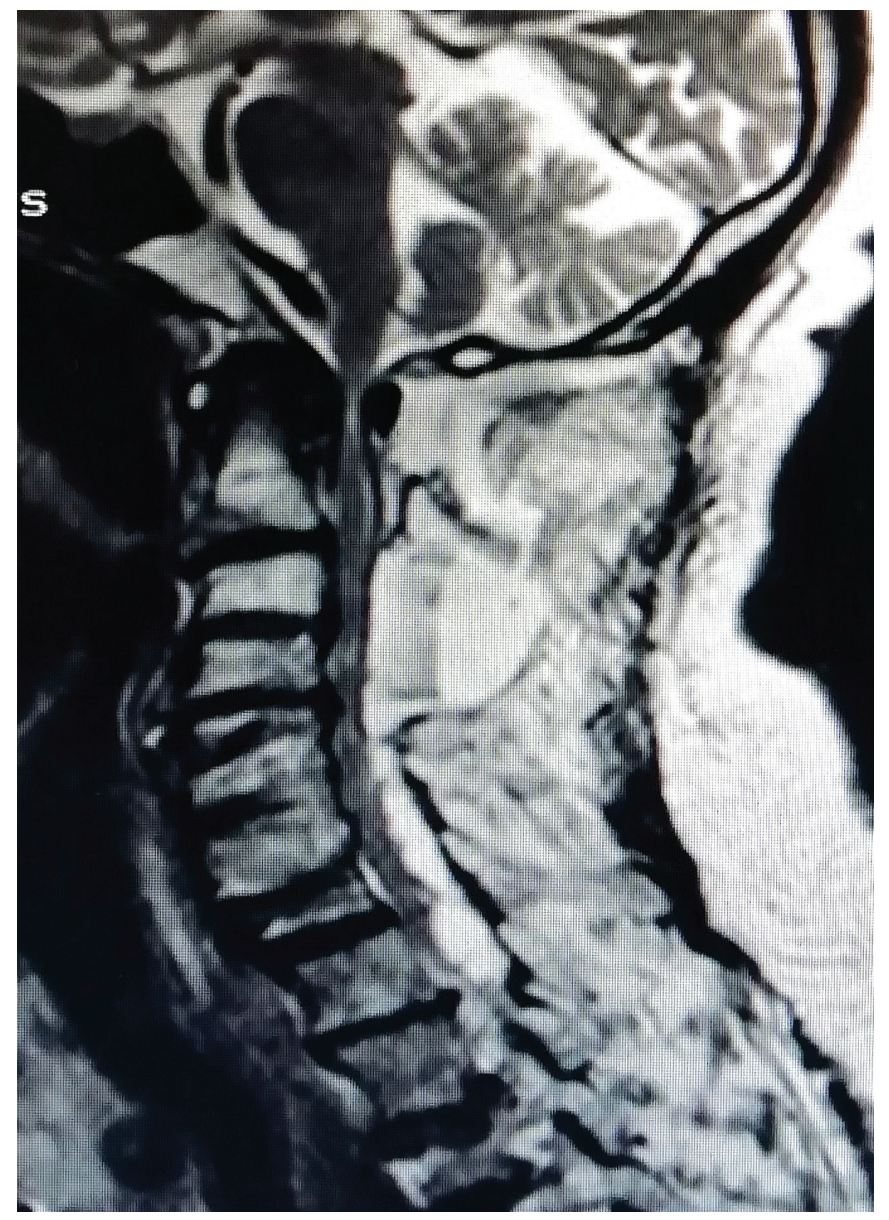

Figure 8. Postoperative sagittal T2-weighted magnetic resonance imaging show the hemorrhage
3-4 dermatome. The complete blood count (hemoglobin, hematocrit, and platelet count), biochemistry profile (kidney function, liver function, proteins, and glucose), prothrombin time, and INR were normal, but he had additional comorbidities (irregular hypertension, coronary artery by-pass surgery, smoking). Drainage system was not present in the surgical area of the patient.According to electroneuromyography, the patient had bilateral C3-T1 segments of anterior forearm, anterior horn and chronic periodic axonal injury. Early period CT revealed pneumorrhachis in the cervical region (Figure 7) and epidural hematoma extending from cervical to thoracic region was detected in MRI (Figure 8-11). We started anti-edema treatment. We planned decompression surgery. The patient and his family refused surgery. Conservative treatment was continued. A highdose steroid treatment was started and continued for 7 days. We started early physiotherapy. Seven days after surgery, there was an improvement in paresis at first lower extremities than upper ones. He could resist gravity in bed. After two weeks, his sensorimotor function recovered. He was able to walk by himself. After 3 weeks, the patient's neurological findings improved and he was discharged.

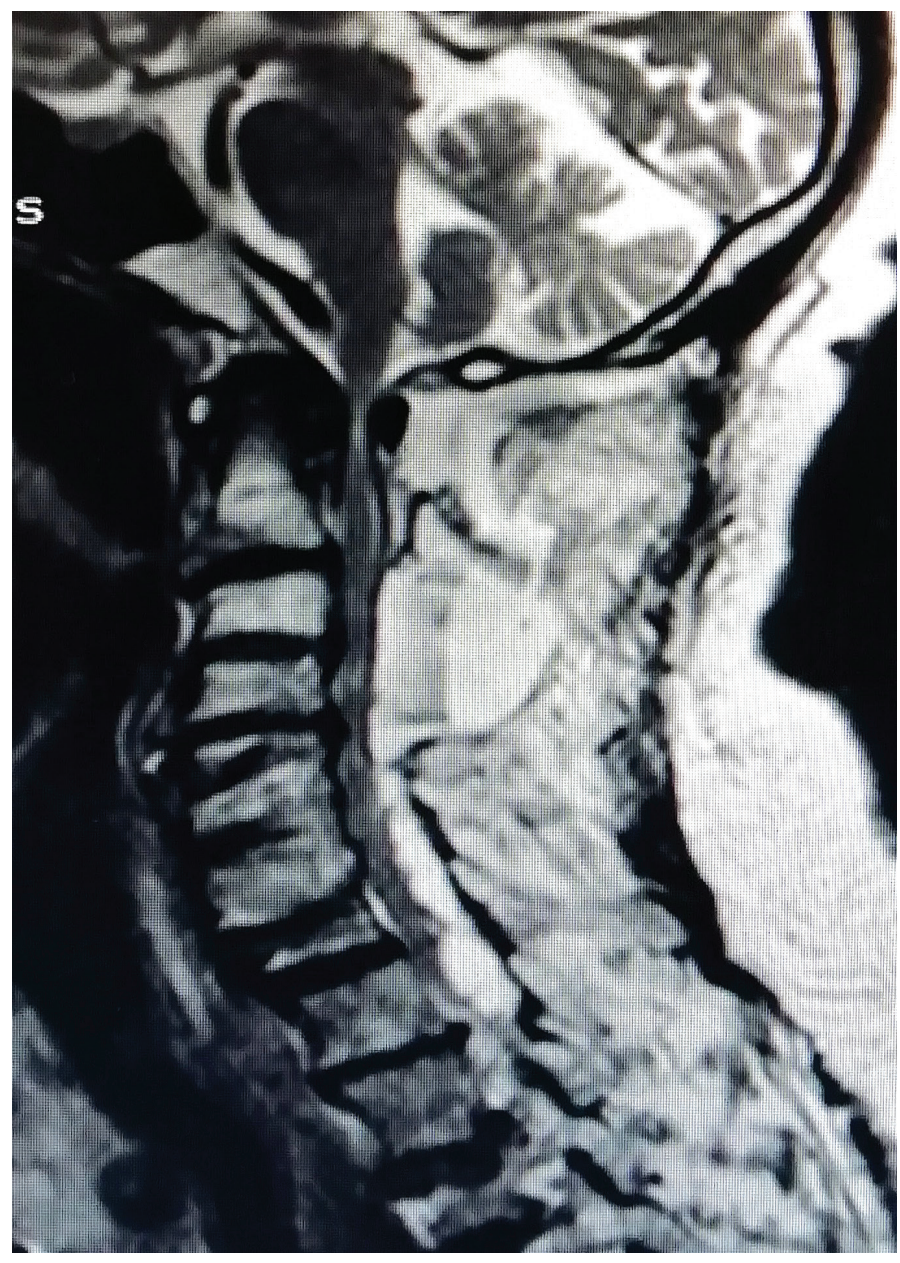

Figure 9. Postoperative cervical axial T2-weighted magnetic resonance imaging 


\section{DISCUSSION}

Total incidence of PSEH was $0.090 \%{ }^{(10)}$. Anterior cervical approach procedures $(0.0563 \%)$ rate is less than posterior ones $(0.13 \%)^{(10)}$. The incidence rate ranged from $0.1 \%$ to $3 \%$,

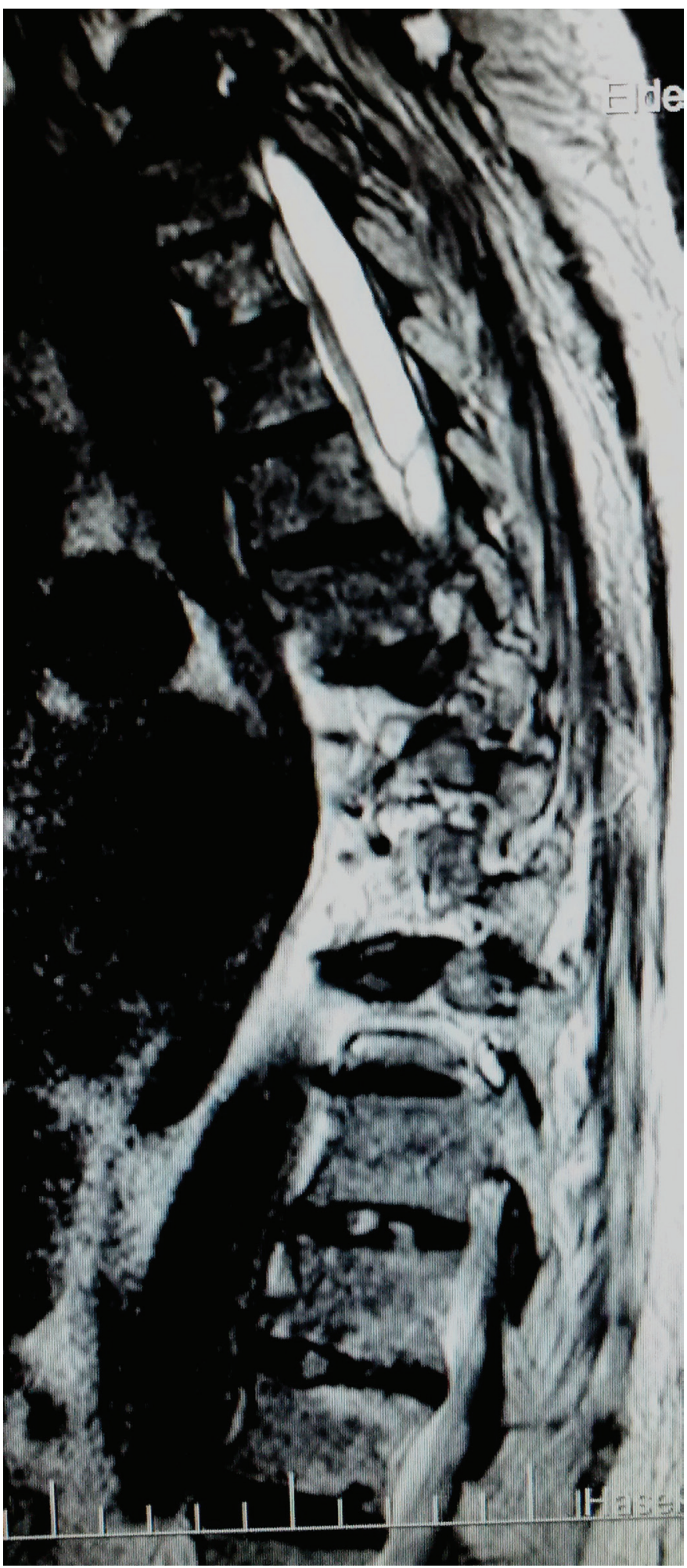

Figure 10. Postoperative thoracic T2-weighted axial magnetic resonance imaging occurring in approximately 1 out of 1000 spinal surgery cases $^{(3,9,10)}$. Acute spinal epidural hematoma is mostly seen at the thoracic spine. At cervical region, epidural hematoma was seen at the highly mobile C6-7 segment $(90 \%)^{(4)}$. However, in opposition to the literature, some published studies suggest that the cervical spine may be the most common region of bleeding ${ }^{(4)}$. Using non-steroidal anti-inflammatory medication, Rh positive blood, elderly patient (above 60 years), pregnancy, preoperative coagulopathy and multilevel surgery (more than 6 levels), greater blood loss, and smoking may be the risk factors $(2,3,8,10)$. The risk of spinal epidural hemorrhage increases with long level surgery and high blood loss. The usage of drainage system reduces the risk of hematoma's mass effect and neurological distress. It reduces the wound complications ${ }^{(9,11)}$. The anticoagulants agents increase the risk of postoperative epidural hematoma. It is recommended to use low-dose heparin instead of acetylsalicylic acid and warfarin sodium before surgery. Four days after stopping acetylsalicylic acid, operation is recommended ${ }^{(3,7)}$. Low-dose heparin use has not been associated with the occurrence of epidural hematoma ${ }^{(3)}$. Both acetylsalicylic acid and non-steroidal anti-inflammatory medications block the cyclooxygenase system, inhibiting platelet aggregation and reducing prostaglandin synthesis. Warfarin sodium acts as an antagonist of vitamin K. Smoking causes thrombosis ${ }^{(8)}$. If there is an acute neurologic regression, absorbable hemostatic agents should always be thought in differential diagnosis ${ }^{(1,11)}$. It usually appears within the first

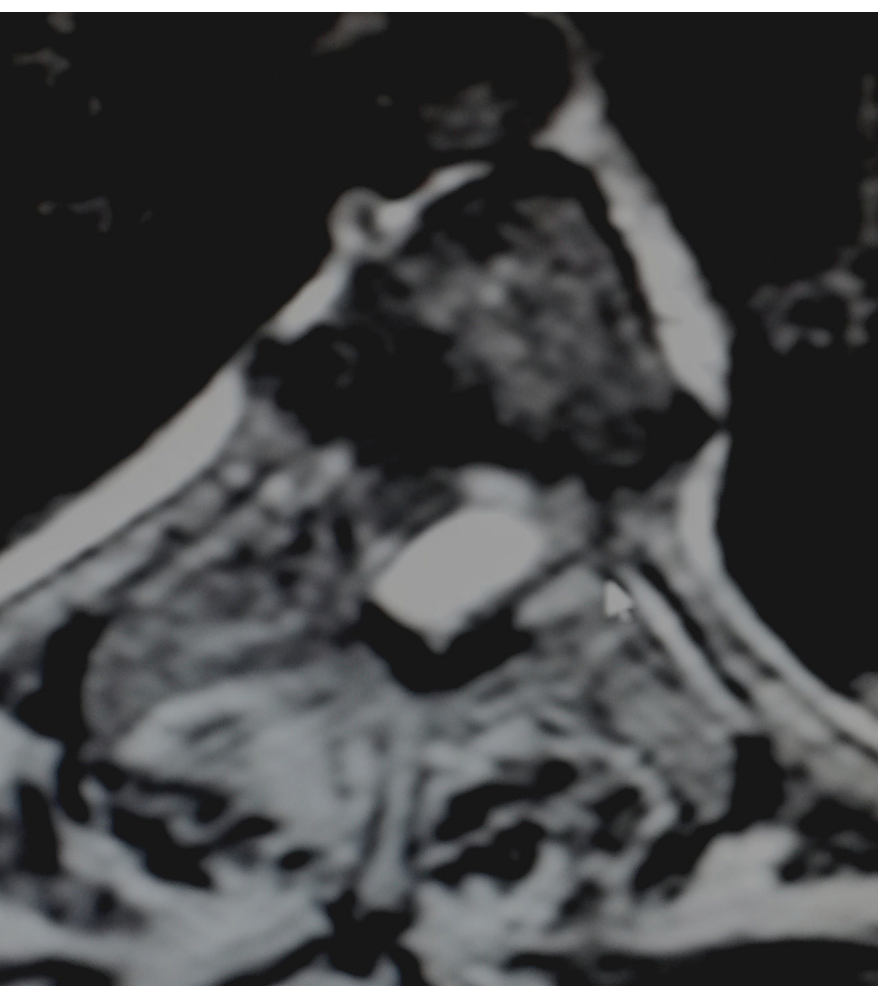

Figure 11. Postoperative thoracic sagittal T2-weighted magnetic resonance imaging 
24 hours. In the late period, the percentage of spinal epidural hematoma (after 4-7 days) was $0.05-0.17 \%^{(2)}$. Neurological impairment occurs within 12 hours and is often followed by rapid progression to complete paralysis ${ }^{(4)}$. After spinal surgery, if there is a neurologic deterioration with new radiologic abnormalities, the literature advises immediate decompressive surgery ${ }^{(5,11)}$. The use of conservative treatment for PSEH with paresis is not favorable at first step treatment management. However, very few reports have discussed the effective nonsurgical management of PSEH ${ }^{(4,5)}$. Here, fortunately the first patient's neurologic deficits improved well in 3 hours. After a high-dose steroid treatment, non-surgical management of PSEH may be a choice in this kind of cases. He had the risk due to smoking and the blood type of Rh $(+)$. In the second patient, there was neurological wellness after 3-week time without surgical decompression. The patient's age was over 60 years. Multilevel spine surgery, blood type of Rh (+), previous history of hypertension and use of acetylsalicylic acid, smoking for more than 40 years, non-use of a drainage system in the operation region were all increased risk factors for spinal epidural hematoma.

PSEH is a rare and dramatic event. It is important to diagnose an epidural hematoma as soon as possible. The major site and common source of bleeding are not clarified. CT scan and MRI are the most accurate methods for precise diagnosis. If neurological deterioration is present, surgery is advised during the first hours. However, conservative management is an alternative pathway in well selected patients with nonprogressive course, but the ethical problem is which patient will have or not. We planned both medical treatment and surgery at the same time. Verifying surgical results and risk factors need to be investigated in larger series.

\section{Ethics}

Informed Consent: All patients signed the free and informed consent form.

Peer-review: Internally peer-reviewed.

\section{Authorship Contributions}

Surgical and Medical Practices: A.A., M.K., Concept: A.A., B.M.K., N.A., Design: A.A., B.M.K., N.A., Data Collection or Processing: A.A., M.K., T.T., Analysis or Interpretation: A.A., M.K., Literature Search: A.A., B.M.K., T.T., Writing: A.A.

Conflict of Interest: No conflict of interest was declared by the authors.

Financial Disclosure: The authors declared that this study received no financial support.

\section{REFERENCES}

1. Akpınar A, Ucler N, Ozdemir CO. Textiloma (gossypiboma) mimicking recurrent intracranial abscess. BMC Res Notes 2015;8:390.

2. Aono H, Ohwada T, Hosono N, Tobimatsu H, Ariga K, Fuji T, et al. Incidence of postoperative symptomatic epidural hematoma in spinal decompression surgery. J Neurosurg Spine 2011;15:202-5.

3. Awad JN, Kebaish KM, Donigan J, Cohen DB, Kostuik JP. Analysis of the risk factors for the development of post-operative spinal epidural hematoma. J Bone Joint Surg Br 2005;87:1248-52.

4. Borges G, Bonilha L, Maldaum MV, Menezes JR, Zanardi V. Acute cervical epidural hematoma: case report. Arq Neuropsiquiatr 2000;58:726-30.

5. Fattahi A, Taheri M. Spontaneous resolved cervical spine epidural hematoma: A case report. Surg Neurol Int 2017;8:183.

6. Koç ER, Acar B, Aytürk Z, Cemil DB, Kösehan D, Illhan A. A case of Spontaneous Spinal Epidural hematoma mimicking Stroke. Turk I Neurol 2014;20:95-7.

7. Korinth MC, Gilsbach JM, Weinzierl MR. Low-dose aspirin before spinal surgery; results of a survey among neurosurgeons in Germany. Eur Spine J 2007;16:365-72.

8. Miao $W, M a X$, Liang $D$, Sun Y. Treatment of hematomas after anterior cervical spine surgery; a retrospective study of 15 cases. Neurochirurgie 2018;64:166-70.

9. Schroeder GD, Kurd MF, Kepler CK, Arnold PM, Vaccaro AR Postoperative epidural hematomas in the Lumbar Spine. J Spinal Disord Tech 2015;28:313-8.

10. Schroeder GD, Hilibrand AS, Arnold PM, Fish DE, Wang JC, Gum JL, et al. Epidural hematoma following cervical spine surgery. Global Spine J 2017;7:120-6.

11. Yi S, Yoon DH, Kim KN, Kim SH, Shin HC. Postoperative Spinal Epidural Hematoma: Risk Factor and Clinical Outcome. Yonsei Med ] 2006;47:326-32. 